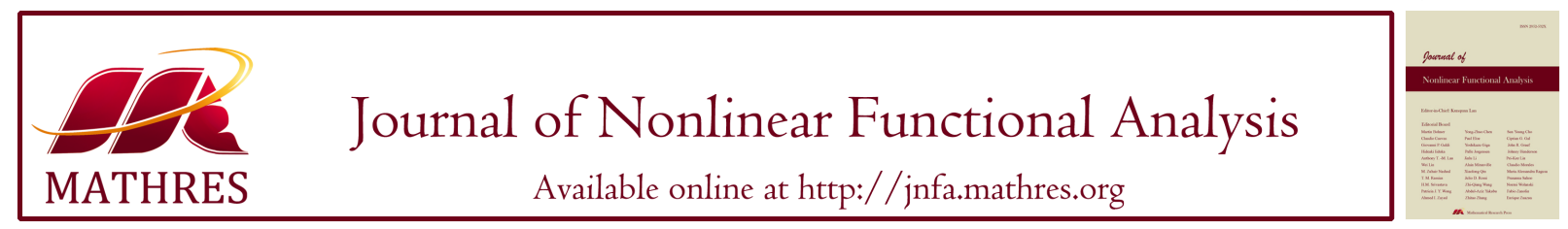

\title{
OPTIMALITY AND DUALITY FOR NONSMOOTH MULTIOBJECTIVE FRACTIONAL PROBLEMS USING CONVEXIFICATORS
}

\author{
DO VAN LUU ${ }^{1,2, *}$, PHAM THI LINH ${ }^{3}$ \\ ${ }^{1}$ TIMAS, Thang Long University, Hanoi, Vietnam \\ ${ }^{2}$ Insitute of Mathematics, Vietnam Academy of Science and Technology, Hanoi, Vietnam \\ ${ }^{3}$ Thai Nguyen University of Economics and Business Administration, Thai Nguyen, Vietnam
}

\begin{abstract}
This paper presents Fritz John necessary conditions for the weak efficiency of multiobjective fractional optimization problems involving equality, inequality and set constraints. With a constraint qualification of Mangasarian-Fromovitz type, Kuhn-Tucker necessary efficiency conditions are established. Under assumptions on generalized convexity, sufficient conditions for weak efficiency are also given together with the theorems of the weak duality, the strong duality, and the inverse duality of Wolfe and Mond-Weir types.
\end{abstract}

Keywords. Multiobjiective fractional problem; Local weak efficient solution; Fritz John and Kuhn-Tucker efficiency conditions; Convexificator; Duality of Wolfe and Mond-Weir types.

\section{INTRODUCTION}

In recent years, many authors studied the optimality conditions for vector optimization problems and obtained Kuhn-Tucker optimality conditions via Lagrange multipliers rules (see, e.g., [1]-[14]). The notion of the nonconvex closed convexificator by Jeyakumar-Luc [15] is a generalization of some notions of known subdifferentials such as the subdifferentials of Clarke [1], Michel-Penot [13], Mordukhovich [14]. It has provided good calculus rules for establishing necessary efficiency conditions in nonsmooth optimization. Necessary conditions for efficiency via convexificators may be sharper than those expressed in terms of known subdifferentials as the Clarke, Michel-Penot and Mordukhovich subdifferentials. For a locally Lipschitz function, which is regular in Clarke's sense, the closed convex hull of the upper regular convexificator is contained in its Clarke subdifferential. This was shown in Example 2.1 by Jeyakumar-Luc [15]. Hence, the optimality conditions in terms of convexifixicators provide sharp results. Luu $[8,9]$ derived necessary conditions for local weak Pareto and Pareto minima of multiobjective

${ }^{*}$ Corresponding author.

E-mail addresses: dvluu @math.ac.vn (D.V. Luu), linhpham19101985@gmail.com (P.T. Linh).

Received September 20, 2020; Accepted December 25, 2020.

(C)2021 Journal of Nonlinear Functional Analysis 
programming problems involving inequality, equality and set constraints in terms of convexificators. Luu [10] established Fritz John and Kuhn-Tucker conditions for local efficient solutions of constrained vector equilibrium problems in Banach spaces in which those solutions are regular in the sense of Ioffe via convexificators. Luu and Mai [11] derived efficiency conditions for multiobjective bilevel programming problems using convexificators.

In various applications of optimization, a function is to be maximized or minimized which involves one or some ratios of functions. Such optimization problems are called fractional programming problems. For example, a related objective is Return per Rist to be maximized, Models where Cost per Time to be maximized, the maximization of Output per Input,.... Liang, Huang and Pardalos [7] derived sufficient optimality conditions and duality for single fractional programming problems. Kuk, Lee and Tanino [6] established necessary and sufficient optimality conditions for nonsmooth multiobjective fractional programming problems with inequality constraints via the Clarke subdifferentials. Gadhi [3] derived optimality conditions for fractional multiobjective problems involving inequality constraints (without equality and set constraints) in terms of convexificators. Chuong [2], Hong, Jiao and Kim [16] studied optimality and duality for fractional robust multiobjective optimization problems.

In this paper, we derive necessary and sufficient efficiency conditions via convexificators for multiobjective fractional problems with equality, inequality and set constraints together with theorems of the weak duality, the strong duality, and the inverse duality of Wolfe and MondWeir types.

The paper is organized as follows. Section 2 gives some notions and preliminaries. Section 3 is devoted to derive Fritz John neceeary optimality conditions for multiobjective fractional problems involving equality, inequality and set constraints via convexificators. Section 4 deals with Kuhn-Tucker necessary conditions for weak efficiency under a constraint qualification of Mangasarian-Fromovitz type. In Section 5, under assumptions on asymptotic pseudoinvexity of the subjective and asymptotic quasiinvexity of constraint, sufficient optimality conditions are given as well. Section 6 gives the theorems of the weak duality, the strong duality, and the inverse duality of Wolfe and Mond-Weir types.

\section{PRELIMINARIES}

Given a Banach space $X$, we denote by $X^{*}$ its topological dual with the canonical dual pairing $\langle.,$.$\rangle , and denote w^{*}$ by the weak* topology on the dual space $X^{*}$. For an extended-real-valued function $f: X \rightarrow \mathbb{R} \cup\{+\infty\}$, the expressions

$$
f^{-}(x ; v)=\liminf _{t \downarrow 0} \frac{f(x+t v)-f(x)}{t},
$$

and

$$
f^{+}(x ; v)=\limsup _{t \downarrow 0} \frac{f(x+t v)-f(x)}{t} .
$$

stand for the lower and upper Dini directional derivatives of $f$ at $x$ in the direction of $v$, respectively.

Let $C \subset \mathbb{R}^{p}$ be a pointed closed convex cone with nonempty interiors, which induces a partial order in $\mathbb{R}^{p}$. Let $A$ be a nonempty subset of $\mathbb{R}^{p}$. A point $\bar{y} \in A$ is said to be a efficient (resp., a 
weak efficient) minimal vector of $A$ with respect to $C$ if

$$
\begin{gathered}
A \subset \bar{y}+\left(\mathbb{R}^{p} \backslash-C\right) \cup\{0\} \\
\left(\text { resp. }, A \subset \bar{y}+\left(\mathbb{R}^{p} \backslash-\operatorname{int}(C)\right),\right.
\end{gathered}
$$

where int denotes the topological interior.

Consider the following multiobjective fractional optimization problem:

$$
(M F P)\left\{\begin{array}{l}
\min \left(\frac{f_{1}(x)}{g_{1}(x)}, \ldots, \frac{f_{p}(x)}{g_{p}(x)}\right), \\
\operatorname{subject~to~} \\
\quad h_{j}(x) \leqslant 0, j \in I:=\{1, \ldots, m\}, \\
\quad \ell_{k}(x)=0, k \in L:=\{1, \ldots r\}, \\
x \in D,
\end{array}\right.
$$

where $f_{i}, g_{i}, h_{j}$ and $\ell_{k}$ are continuous functions defined on $X$ such that $g_{i}(x)>0$, for all $i, j, k$ and all $x \in X, D$ is a closed subset of $X$. Denote by $M$ the feasible set of (MFP)

$$
M:=\left\{x \in D: h_{j}(x) \leq 0, j=1, \ldots, m, \text { and } \ell_{k}(x)=0, k=1, \ldots n\right\},
$$

and for $\bar{x} \in M, I(\bar{x}):=\left\{j \in I: h_{j}(\bar{x})=0\right\}$.

We set $\phi(x):=\left(\frac{f_{1}(x)}{g_{1}(x)}, \ldots, \frac{f_{p}(x)}{g_{p}(x)}\right)$. A point $\bar{x} \in M$ is called an efficient (resp., weak efficient) solution of (MFP) if $\phi(\bar{x})$ is an efficient (resp., weak efficient) minimal vector of $\phi(M)$.

A point $\bar{x} \in M$ is a local efficient (resp., weak local efficient) solution of (MFP) if there exists a neighborhood $V$ of $\bar{x}$ such that $\phi(\bar{x})$ is a Pareto (resp., weak Pareto) minimal vector of $\phi(M \cap V)$.

Recall [17] that a point $\bar{x}$ is said to be a regular point in the sense of Ioffe for $\ell$ relative to $D$ if there exist numbers $K>0$ and $\delta>0$ such that, for all $x \in D \cap B(\bar{x} ; \delta)$,

$$
d(x, Q) \leqslant K\|\ell(x)-\ell(\bar{x})\|,
$$

where $Q=\{x \in D: \ell(x)=\ell(\bar{x})\}, d(x, Q)$ denotes the distance from $x$ to $Q, B(\bar{x} ; \delta)$ stands for the open ball of radius $\delta$ around $\bar{x}$ (see [10]).

Note that for Problem (MFP) without equality constraint, a local weak efficient solution of (MFP) is also a regular point in the sense of Ioffe.

Recall [15] that the funtion $f: X \rightarrow \mathbb{R} \cup\{+\infty\}$ is said to have a convexificator $\partial^{*} f(x)$ at $x$ if $\partial^{*} f(x)\left(\subset X^{*}\right)$ is weak* closed, and, for each $v \in X$,

$$
f^{-}(x, v) \leqslant \sup _{x^{*} \in \partial^{*} f(x)}\left\langle x^{*}, v\right\rangle, \text { and } f^{+}(x, v) \geqslant \inf _{x^{*} \in \partial^{*} f(x)}\left\langle x^{*}, v\right\rangle .
$$

Note that convexificators are not necessarily weak* compact or convex. These relaxations allow applications to a large class of nonsmooth continuous functions. For instance, the function $f: \mathbb{R} \rightarrow \mathbb{R}$ defined by

$$
f(x)= \begin{cases}\sqrt{x}, & \text { if } x \geqslant 0, \\ -\sqrt{-x}, & \text { if } x<0,\end{cases}
$$

admits noncompact convexificators at 0 of the form $[\alpha, \infty)$ with $\alpha \in \mathbb{R}$. 
Remark 2.1. The convex hull of a convexificator of a locally Lipschitz funtion may be strictly contained in both the Clarke and Michel-Penot subdiffirentials (see [15]). Hence, necessary optimality conditions that are expressed in terms of $\partial^{*} f(x)$ may provide sharp conditions even for locally Lipschitz funtions. For example,

$$
f(x)= \begin{cases}x^{2} \sin \frac{1}{x}, & x \neq 0, \\ 0, & x=0,\end{cases}
$$

Then $\partial^{C} f(0)=\partial^{M P} f(0)=[-1,1]$ (Example 2.2.3 in [1]), where $\partial^{C}$ and $\partial^{M P}$ indicate the Clarke and Michel-Penot subdifferentials, respectively. The set $\{-1 ; 1\}$ is a convexificator of $f$ at $\bar{x}=0$. Then, $\{-1 ; 1\} \varsubsetneqq[-1,1]$.

The chain rule for composite functions in [15] is needed in the following.

Proposition 2.2. [15] Let $f=\left(f_{1}, \ldots, f_{n}\right)$ be a continuous function from $X$ to $\mathbb{R}^{n}$, and let $g$ be a continuous function from $\mathbb{R}^{n}$ to $\mathbb{R}$. Suppose that, for each $i=1, \ldots, n, f_{i}$ admits a bounded convexificator $\partial^{*} f_{i}(\bar{x})$ at $\bar{x}$ and that $g$ admits a bounded convexificator $\partial^{*} g(f(\bar{x}))$ at $f(\bar{x})$. For each $i=1, \ldots, n$, if $\partial^{*} f_{i}$ is upper semicontinuous at $\bar{x}$ and $\partial^{*} g$ is upper semicontinuous at $f(\bar{x})$, then

$$
\partial^{*} g(f(\bar{x}))\left(\partial^{*} f_{1}(\bar{x}), \ldots, \partial^{*} f_{n}(\bar{x})\right)
$$

is a convexificator of $g \circ f$ at $\bar{x}$.

For a set $D \subset X$, the Clarke tangent cone to $D$ at $\bar{x}$ is defined as

$$
\begin{aligned}
T(D ; \bar{x})= & \left\{v \in X: \forall x_{n} \in D, x_{n} \rightarrow \bar{x}, \forall t_{n} \downarrow 0, \exists v_{n} \rightarrow v\right. \\
& \text { such that } \left.x_{n}+t_{n} v_{n} \in D, \forall n\right\} .
\end{aligned}
$$

The Clarke normal cone to $D$ at $\bar{x}$ is

$$
N(D ; \bar{x})=\left\{\xi \in X^{*}:\langle\xi, v\rangle \leqslant 0, \forall v \in T(D ; \bar{x})\right\} .
$$

\section{Fritz JOHN NECESSARY EFFICIENCY CONDITIONS}

To derive Fritz John necessary efficiency conditions for (MFP), we introduce the following Assumption.

Assumption 3.1. $\quad$ (i) $f_{i}, g_{i}, h_{j}, \ell_{k}$ are continuous and admit convexificators $\partial^{*} f_{i}(x), \partial^{*} g_{i}(x)$, $\partial^{*} h_{j}(x), \partial^{*} \ell_{k}(x)$ at $x$ near $\bar{x}$, respectively. They admit bounded convexificators $\partial^{*} f_{i}(\bar{x})$, $\partial^{*} g_{i}(\bar{x}), \partial^{*} h_{j}(\bar{x}), \partial^{*} \ell_{k}(\bar{x})$ at $\bar{x}$. Moreover, $\partial^{*} f_{i}, \partial^{*} g_{i}, \partial^{*} h_{j}, \partial^{*} \ell_{k}$ are upper semicontinuous at $\bar{x} ; D$ is convex;

(ii) $\ell_{1}, \ldots, \ell_{r}$ are locally Lipschitz at $\bar{x} ;\left|\ell_{k}\right|(k \in L)$ are regular in the sense of Clarke.

Remark 3.2. Assumption 3.1 together with the fact that $\bar{x}$ is a regular point in the sense of Ioffe for $\ell$ relative to $D$ ensures that all the hypotheses of Theorem 6.4 [10] are fulfilled.

We now begin with a Fritz John necessary efficiency condition for (MFP).

Theorem 3.3. Let $\bar{x} \in M$ be a local weak efficient solution of $(M F P)$. Suppose that $\bar{x}$ is a regular point in the sense of Ioffe for $\ell$ relative to $D$. Assume, furthermore, that Assumption 
3.1 is fulfilled. Then there exists vector $\left(\lambda_{1}^{*}, \ldots, \lambda_{p}^{*}\right) \in \mathbb{R}_{+}^{p} \backslash\{0\},\left(\mu_{0}^{*}, \mu_{1}^{*}, \ldots, \mu_{m}^{*}\right) \in \mathbb{R}_{+}^{m+1}$, and $\left(v_{1}^{*}, \ldots, v_{r}^{*}\right) \in \mathbb{R}^{r}$ such that

$$
\begin{gathered}
0 \in \operatorname{cl}\left(\mu_{0}^{*} \sum_{i=1}^{p} \lambda_{i}^{*}\left(\operatorname{conv} \partial^{*} f_{i}(\bar{x})-\phi_{i}(\bar{x}) \operatorname{conv} \partial^{*} g_{i}(\bar{x})\right)\right. \\
\left.+\sum_{j=1}^{m} \mu_{j}^{*} \operatorname{conv} \partial^{*} h_{j}(\bar{x})+\sum_{k=1}^{r} v_{k}^{*} \operatorname{conv} \partial^{*} \ell_{k}(\bar{x})+N(D ; \bar{x})\right) \\
\mu_{0}^{*}+\sum_{j=1}^{m} \mu_{j}^{*}=1, \\
\mu_{j}^{*} h_{j}(\bar{x})=0, j=1, \ldots, m,
\end{gathered}
$$

where cl indicates the weak* closure, conv stands for the convex hull.

Proof. Since $\bar{x} \in M$ is a local weak efficient solution of (MFP), there exists a neighborhood $U$ of $\bar{x}$ such that

for all $x \in U \cap M$, where $\mathbb{R}_{++}^{p}=$ int $\mathbb{R}_{+}^{p}$.

$$
\phi(x)-\phi(\bar{x}) \in \mathbb{R}^{p} \backslash-\mathbb{R}_{++}^{p},
$$

Let us prove that $\bar{x}$ is a local weak minimal solution of the following problem:

$$
\left(P_{1}\right):\left\{\begin{array}{l}
\min \left(f_{1}(x)-\phi_{1}(\bar{x}) g_{1}(x), \ldots, f_{p}(x)-\phi_{p}(\bar{x}) g_{p}(x)\right), \\
\text { subject to } \\
x \in M,
\end{array}\right.
$$

where $\phi_{i}(\bar{x})=\frac{f_{i}(\bar{x})}{g_{i}(\bar{x})}(i=1, \ldots, p)$. If not, there exists $x_{1} \in U \cap M$ such that

$$
\left(f_{i}\left(x_{1}\right)-\phi_{i}(\bar{x}) g_{i}\left(x_{1}\right)\right)-\left(f_{i}(\bar{x})-\phi_{i}(\bar{x}) g_{i}(\bar{x})\right) \in-\mathbb{R}_{++}(i=1, \ldots, p) .
$$

Since $f_{i}(\bar{x})-\phi_{i}(\bar{x}) g_{i}(\bar{x})=0$, one has

$$
\frac{f_{i}\left(x_{1}\right)}{g_{i}\left(x_{1}\right)}-\frac{f_{i}(\bar{x})}{g_{i}(\bar{x})} \in-\mathbb{R}_{++}(i=1, \ldots, p) .
$$

Hence,

$$
\phi\left(x_{1}\right)-\phi(\bar{x}) \in \mathbb{R}_{++}^{p},
$$

which contradicts (3.4). Applying a scalarization theorem by Gong ([4], Theorem 3.1) to Problem $\left(P_{1}\right)$ yields the existence of a continuous positively homogeneous subadditive function $\Lambda$ on $X$ satisfying

$$
y_{2}-y_{1} \in \mathbb{R}_{++}^{p} \Longrightarrow \Lambda\left(y_{1}\right)<\Lambda\left(y_{2}\right),
$$

and

$$
\Lambda(\varphi(x)-\varphi(\bar{x})) \geqslant 0(\forall x \in U \cap M),
$$

where $\varphi_{i}(x):=f_{i}(x)-\phi_{i}(\bar{x}) g_{i}(x), i \in\{1, \ldots, p\}$, and $\varphi=\left(\varphi_{1}, \ldots, \varphi_{p}\right)$. Since $\varphi(\bar{x})=0$ and $\Lambda$ is positively homogeneous, it follows from (3.5) that

$$
\Lambda(\varphi(x)) \geqslant 0=\Lambda(\varphi(\bar{x})),
$$

which means that $\bar{x}$ is a local minimal solution of the following scalar problem:

$$
\left(P_{2}\right):\left\{\begin{array}{l}
\text { minimize } \Lambda\left(\varphi_{1}(x)-\varphi_{1}(\bar{x}), \ldots, \varphi_{p}(x)-\varphi_{p}(\bar{x})\right), \\
\text { subject to } x \in M
\end{array}\right.
$$


It should be noted here that all the hypotheses of Theorem 6.4 [10] are fulfilled. We invoke this theorem to deduce that there exist $\mu_{0}^{*} \geqslant 0, \mu_{j}^{*} \geqslant 0, j=1, \ldots, m, v_{k}^{*} \in \mathbb{R}, k=1, \ldots, r$ such that

$$
\mu_{0}^{*}+\sum_{i=1}^{m} \mu_{j}^{*}=1,
$$

and

$$
\begin{aligned}
0 \in \operatorname{cl}\left(\mu_{0}^{*} \operatorname{conv} \partial^{*} \Lambda\left(\varphi_{1}-\varphi_{1}(\bar{x}), \ldots, \varphi_{p}-\varphi_{p}(\bar{x})\right)(\bar{x})\right. \\
\left.\quad+\sum_{j \in I(\bar{x})} \mu_{j}^{*} \operatorname{conv} \partial^{*} h_{j}(\bar{x})+\sum_{k=1}^{r} v_{k}^{*} \operatorname{conv} \partial^{*} \ell_{k}(\bar{x})+N(D ; \bar{x})\right)
\end{aligned}
$$

which is equivalent to the following

$$
\begin{gathered}
0 \in \operatorname{cl}\left(\mu_{0}^{*} \operatorname{conv} \partial^{*} \Lambda\left(\varphi_{1}(.)-\varphi_{1}(\bar{x}), \ldots, \varphi_{p}(.)-\varphi_{p}(\bar{x})\right)(\bar{x})+\sum_{j=1}^{m} \mu_{j}^{*} \operatorname{conv} \partial^{*} h_{j}(\bar{x})\right. \\
\left.+\sum_{k=1}^{r} v_{k}^{*} \operatorname{conv} \partial^{*} \ell_{k}(\bar{x})+N(D ; \bar{x})\right) \\
\mu_{j}^{*} h_{j}(\bar{x})=0, j=1, \ldots, m .
\end{gathered}
$$

It can be seen that $\Lambda$ is a continuous convex function on $\mathbb{R}^{p}$, and hence, it is locally Lipschitz on $\mathbb{R}^{p}$. Hence, we can choose the convex subdifferential $\partial \Lambda(0)$ as a convexificator of $\Lambda$ at 0 . Then $\partial \Lambda(0)$ is a bounded convexificator of $\Lambda$ at

$$
\left(\varphi_{1}(\bar{x})-\varphi_{1}(\bar{x}), \ldots, \varphi_{p}(\bar{x})-\varphi_{p}(\bar{x})\right)=(0, \ldots, 0) .
$$

Moreover, the mapping $\partial \Lambda$ is upper semicontinuous at $(0, \ldots, 0)$ since $\Lambda$ is locally Lipschitz on $\mathbb{R}^{p}$. Taking account of Proposition 2.2 to the composite function $\Lambda\left(\varphi_{1}-\varphi_{1}(\bar{x}), \ldots, \varphi_{p}-\right.$ $\left.\varphi_{p}(\bar{x})\right)($.$) , one gets that the set \partial \Lambda(0)\left(\partial^{*} \varphi_{1}(\bar{x}), \ldots, \partial^{*} \varphi_{p}(\bar{x})\right)$ is a convexificator of $\Lambda\left(\varphi_{1}-\right.$ $\left.\varphi_{1}(\bar{x}), \ldots, \varphi_{p}-\varphi_{p}(\bar{x})\right)($.$) at \bar{x}$, where $\partial \Lambda(0)$ is the convex subdifferential of $\Lambda$ at 0 . Note that $\partial^{*} f_{i}(\bar{x})-\phi_{i}(\bar{x}) \partial^{*} g_{i}(\bar{x})$ is a convexificator of the function $\varphi_{i}:=f_{i}-\phi_{i}(\bar{x}) g_{i}$ at $\bar{x}(i=1, \ldots, p)$. In fact, for $i=1, \ldots, p$, we set

$$
\begin{gathered}
G\left(u_{i}, v_{i}\right):=u_{i}-\phi_{i}(\bar{x}) v_{i}, \\
F(x):=\left(f_{i}(x), g_{i}(x)\right) .
\end{gathered}
$$

Then, $G$ is Fréchet differentiable at $\bar{x}$, and

$$
\partial^{*} G(F(\bar{x}))=\left\{\left(1,-\phi_{i}(\bar{x})\right)\right\} .
$$

Taking account of Proposition 2.2, it follows that the following set is a convexificator of $G \circ F$ at $\bar{x}$

$$
\left.\left.\left(1,-\phi_{i}(\bar{x})\right)\right)\left(\left(\partial^{*} f_{i}(\bar{x}), \partial^{*} g_{i}(\bar{x})\right)\right)=\partial^{*} f_{i}(\bar{x})-\phi_{i}(\bar{x}) \partial^{*} g_{i}(\bar{x})\right)
$$

In view of (3.6), one gets

$$
\begin{aligned}
0 \in \operatorname{cl}\left(\mu_{0}^{*} \partial \Lambda(0)\left(\operatorname{conv} \partial^{*} \varphi_{1}(\bar{x}), \ldots, \operatorname{conv} \partial^{*} \varphi_{p}(\bar{x})\right)\right. \\
\left.+\sum_{j=1}^{m} \mu_{j}^{*} \operatorname{conv} \partial^{*} h_{j}(\bar{x})+\sum_{k=1}^{r} v_{k}^{*} \operatorname{conv} \partial^{*} \ell_{k}(\bar{x})+N(D ; \bar{x})\right) .
\end{aligned}
$$


Hence, the above inclusion implies that there exists a sequence

$$
\begin{aligned}
\theta_{n} \in \mu_{0}^{*} \partial \Lambda(0)\left(\operatorname{conv} \partial^{*} \varphi_{1}(\bar{x}), \ldots, \operatorname{conv} \partial^{*} \varphi_{p}(\bar{x})\right) \\
+\sum_{j=1}^{m} \mu_{j}^{*} \operatorname{conv} \partial^{*} h_{j}(\bar{x})+\sum_{k=1}^{r} v_{k}^{*} \operatorname{conv} \partial^{*} \ell_{k}(\bar{x})+N(D ; \bar{x})
\end{aligned}
$$

such that $\lim _{n \rightarrow \infty} \theta_{n}=0$. The latter implies that there exists a sequence $\left\{\lambda_{n}\right\} \subset \partial \Lambda(0)$ such that

$$
\begin{aligned}
\theta_{n} \in \mu_{0}^{*} \lambda_{n}\left(\operatorname{conv} \partial^{*} \varphi_{1}(\bar{x}), \ldots, \operatorname{conv} \partial^{*} \varphi_{p}(\bar{x})\right) \\
+\sum_{j=1}^{m} \mu_{j}^{*} \operatorname{conv} \partial^{*} h_{j}(\bar{x})+\sum_{k=1}^{r} v_{k}^{*} \operatorname{conv} \partial^{*} \ell_{k}(\bar{x})+N(D ; \bar{x}) .
\end{aligned}
$$

Since $\partial \Lambda(0)$ is a compact set in $\mathbb{R}^{p}$, without loss of generality, we can suppose that $\lambda_{n} \rightarrow \lambda^{*} \in$ $\partial \Lambda(0)$. By letting $n \rightarrow \infty$, we get that

$$
\begin{aligned}
0 \in \operatorname{cl}\left(\mu_{0}^{*} \lambda^{*}\left(\operatorname{conv} \partial^{*} \varphi_{1}(\bar{x}), \ldots, \operatorname{conv} \partial^{*} \varphi_{p}(\bar{x})\right)\right. \\
+\sum_{j=1}^{m} \mu_{j}^{*} \operatorname{conv} \partial^{*} h_{j}(\bar{x})+\sum_{k=1}^{r} v_{k}^{*} \operatorname{conv} \partial^{*} \ell_{k}(\bar{x})+N(D ; \bar{x}) .
\end{aligned}
$$

Let us show that $\lambda^{*} \in \mathbb{R}^{p} \backslash\{0\}$. In fact, for $y \in \mathbb{R}_{++}^{p}$, it can be written as $0-(-y) \in \mathbb{R}_{++}^{p}$. Therefore,

$$
\begin{aligned}
\left\langle\lambda^{*},-y\right\rangle & =\left\langle\lambda^{*},-y-0\right\rangle \\
& \leqslant \Lambda(-y)-\Lambda(0) \\
& =\Lambda(-y)<\Lambda(0)=0 .
\end{aligned}
$$

Consequently, $\lambda^{*} \in \mathbb{R}_{+}^{p} \backslash\{0\}$. Hence, $\lambda^{*}=\left(\lambda_{1}^{*}, \ldots, \lambda_{p}^{*}\right) \in \mathbb{R}_{+}^{p} \backslash\{0\}$, and

$$
\begin{aligned}
0 \in \operatorname{cl}\left(\mu_{0}^{*}\left(\lambda_{1}^{*}, \ldots, \lambda_{p}^{*}\right)\left(\operatorname{conv} \partial^{*} \varphi_{1}(\bar{x}), \ldots, \operatorname{conv} \partial^{*} \varphi_{p}(\bar{x})\right)\right. \\
+\sum_{j=1}^{m} \mu_{j}^{*} \operatorname{conv} \partial^{*} h_{j}(\bar{x})+\sum_{k=1}^{r} v_{k}^{*} \operatorname{conv} \partial^{*} \ell_{k}(\bar{x})+N(D ; \bar{x}) .
\end{aligned}
$$

It follows that

$$
\begin{aligned}
0 \in \operatorname{cl}\left(\mu_{0}^{*} \sum_{i=1}^{p} \lambda_{i}^{*}\left(\operatorname{conv} \partial^{*} f_{i}(\bar{x})-\phi_{i}(\bar{x}) \operatorname{conv} \partial^{*} g_{i}(\bar{x})\right)\right. \\
\left.+\sum_{j=1}^{m} \mu_{j}^{*} \operatorname{conv} \partial^{*} h_{j}(\bar{x})+\sum_{k=1}^{r} v_{k}^{*} \operatorname{conv} \partial^{*} \ell_{k}(\bar{x})+N(D ; \bar{x})\right) .
\end{aligned}
$$

The proof is complete.

\section{Kuhn-Tucker Necessary EfFiciency Conditions}

To derive Kuhn-Tucker necessary conditions, we introduce the following MangasarianFromovitz type constraint qualification $(C Q)$ for multiobjective fractional programming problem (MFP): There exist $v_{0} \in T(D ; \bar{x})$ and numbers $a_{i}>0(i \in I(\bar{x}))$ such that

(i) $\left\langle\xi, v_{0}\right\rangle \leqslant-a_{i}\left(\forall \xi \in \operatorname{conv} \partial^{*} h_{i}(\bar{x}), \forall i \in I(\bar{x})\right)$;

(ii) $\left\langle\eta_{j}, v_{0}\right\rangle=0\left(\forall \eta_{j} \in \operatorname{conv} \partial^{*} \ell(\bar{x}), \forall j \in\{1, \ldots, n\}\right)$. 
A Kuhn-Tucker necessary efficiency condition for (MFP) can be stated as follows.

Theorem 4.1. Let $\bar{x} \in M$ be a local weak efficient solution of (MFP), and let all the hypotheses of Theorem 3.3 be fufilled. Assume furthermore the constraint qualification $(C Q)$ holds. Then, there exists vector $\left(\alpha_{1}^{*}, \ldots, \alpha_{p}^{*}\right) \in \mathbb{R}_{+}^{p} \backslash\{0\},\left(\mu_{1}^{*}, \ldots, \mu_{m}^{*}\right) \in \mathbb{R}_{+}^{m}$, and $\left(v_{1}^{*}, \ldots, v_{r}^{*}\right) \in \mathbb{R}^{r}$ such that

$$
\begin{gathered}
0 \in \operatorname{cl}\left(\sum_{i=1}^{p} \alpha_{i}^{*}\left(\operatorname{conv} \partial^{*} f_{i}(\bar{x})-\phi_{i}(\bar{x}) \operatorname{conv} \partial^{*} g_{i}(\bar{x})\right)\right. \\
\left.+\sum_{j=1}^{m} \mu_{j}^{*} \operatorname{conv} \partial^{*} h_{j}(\bar{x})+\sum_{k=1}^{r} v_{k}^{*} \operatorname{conv} \partial^{*} \ell_{k}(\bar{x})+N(D ; \bar{x})\right), \\
\mu_{j}^{*} h_{j}(\bar{x})=0, j=1, \ldots, m .
\end{gathered}
$$

Proof. Under the Assumptions of Theorem 3.3, we claim that there exists vector $\left(\lambda_{1}^{*}, \ldots, \lambda_{p}^{*}\right) \in$ $\mathbb{R}_{+}^{p} \backslash\{0\},\left(\mu_{0}^{*}, \mu_{1}^{*}, \ldots, \mu_{m}^{*}\right) \in \mathbb{R}_{+}^{m+1}$, and $\left(v_{1}^{*}, \ldots, v_{r}^{*}\right) \in \mathbb{R}^{r}$ such that

$$
\begin{gathered}
0 \in \operatorname{cl}\left(\mu_{0}^{*} \sum_{i=1}^{p} \lambda_{i}^{*}\left(\operatorname{conv} \partial^{*} f_{i}(\bar{x})-\phi_{i}(\bar{x}) \operatorname{conv} \partial^{*} g_{i}(\bar{x})\right)\right. \\
\left.+\sum_{j=1}^{m} \mu_{j}^{*} \operatorname{conv} \partial^{*} h_{j}(\bar{x})+\sum_{k=1}^{r} v_{k}^{*} \operatorname{conv} \partial^{*} \ell_{k}(\bar{x})+N(D ; \bar{x})\right) \\
\mu_{0}^{*}+\sum_{j=1}^{m} \mu_{j}^{*}=1, \\
\mu_{j}^{*} h_{j}(\bar{x})=0, j=1, \ldots, m .
\end{gathered}
$$

If $\mu_{0}^{*}=0$, then $\sum_{j=1}^{m} \mu_{j}^{*}=1$. By virtue of (4.3), we have that there exist $\xi_{j}^{(n)} \in \operatorname{conv} \partial^{*} h_{j}(\bar{x})$, $\eta_{k}^{(n)} \in \operatorname{conv} \partial^{*} \ell_{k}(\bar{x})$ and $\zeta^{(n)} \in N(C ; \bar{x})$ such that

$$
\lim _{n \rightarrow \infty}\left[\sum_{j \in I(\bar{x})} \mu_{j}^{*} \xi_{j}^{(n)}+\sum_{k \in L} v_{k}^{*} \eta_{k}^{(n)}+\zeta^{(n)}\right]=0 .
$$

Hence,

$$
\lim _{n \rightarrow \infty}\left[\sum_{j \in I(\bar{x})} \mu_{j}^{*}\left\langle\xi_{j}^{(n)}, v_{0}\right\rangle+\sum_{k \in L} v_{k}^{*}\left\langle\eta_{k}^{(n)}, v_{0}\right\rangle+\left\langle\zeta^{(n)}, v_{0}\right\rangle\right]=0
$$

where $v_{0} \in T\left(C ; x_{0}\right)$ is available in the constraint qualification (CQ).

On the other hand, since $\mu_{i}^{*} \geqslant 0, i=1, \ldots, m, \sum_{j=1}^{m} \mu_{j}^{*}=1$, we have

$$
\begin{aligned}
0 & =\lim _{n \rightarrow \infty}\left[\sum_{j \in I(\bar{x})} \mu_{j}^{*}\left\langle\xi_{j}^{(n)}, v_{0}\right\rangle+\sum_{k \in L} v_{k}^{*}\left\langle\eta_{k}^{(n)}, v_{0}\right\rangle+\left\langle\zeta^{(n)}, v_{0}\right\rangle\right] \\
& \leqslant \lim _{n \rightarrow \infty}\left[\sum_{j \in I(\bar{x})} \mu_{j}^{*}\left\langle\xi_{j}^{(n)}, v_{0}\right\rangle+\sum_{k \in L} v_{k}^{*}\left\langle\eta_{k}^{(n)}, v_{0}\right\rangle\right] \\
& \leqslant-\sum_{i \in I(\bar{x})} \mu_{j}^{*} a_{j}<0,
\end{aligned}
$$


a contradiction(!). Hence, $\mu_{0}>0$. We set $\alpha_{i}^{*}=\mu_{0}^{*} \lambda_{i}^{*}(i=1, \ldots, p)$, we obtain $\left(\alpha_{1}^{*}, \ldots, \alpha_{p}^{*}\right) \in$ $\mathbb{R}_{+}^{m} \backslash\{0\}$, and

$$
\begin{aligned}
0 \in \operatorname{cl}\left(\sum_{i=1}^{p} \alpha_{i}^{*}\right. & \left(\operatorname{conv} \partial^{*} f_{i}(\bar{x})-\phi_{i}(\bar{x}) \operatorname{conv} \partial^{*} g_{i}(\bar{x})\right) \\
& \left.+\sum_{j=1}^{p} \mu_{j}^{*} \operatorname{conv} \partial^{*} h_{j}(\bar{x})+\sum_{k=1}^{r} v_{k}^{*} \operatorname{conv} \partial^{*} \ell_{k}(\bar{x})+N(D ; \bar{x})\right)
\end{aligned}
$$

This completes the proof.

Theorem 4.1 is illustrated by the following example.

Example 4.2. Let $X=\mathbb{R}^{2}$ and $D=[0,1] \times[0,1]$. Give the following functions:

$$
\begin{aligned}
& f_{1}(x)=\left|x_{1}\right|-x_{2}+1, \\
& g_{1}(x)=-x_{2}+1, \\
& f_{2}(x)=x_{1}^{2}\left(\sin \frac{1}{x_{1}}-1\right), \\
& g_{2}(x)=e^{-x_{2}}, \\
& f_{3}(x):=\left\{\begin{array}{l}
\frac{x_{1}}{1+e^{\frac{1}{x_{1}}}}, \quad \text { if } \quad x_{1} \neq 0, \\
0,
\end{array} ;\right. \\
& g_{3}(x)=-x_{2}+2, \\
& h_{1}(x)=x_{1}\left(x_{1}-1\right), \\
& h_{2}(x)=x_{1}-\left|x_{1}\right|, \\
& \ell(x)=x_{2}-2 x_{1} .
\end{aligned}
$$

Consider the following multiobjective fractional programming problem:

$$
\begin{aligned}
& \min \left(\frac{f_{1}(x)}{g_{1}(x)}, \frac{f_{2}(x)}{g_{2}(x)}, \frac{f_{3}(x)}{g_{3}(x)}\right), \\
& \text { s.t. } \quad h_{i}(x) \leqslant 0(i=1,2), \\
& \ell(x)=0, \\
& x \in D .
\end{aligned}
$$

The feasible set of $\left(P_{3}\right)$ is

$$
\begin{aligned}
M & =\left\{x=\left(x_{1}, x_{2}\right) \in D: h_{1}(x) \leqslant 0, h_{2}(x) \leqslant 0, \ell(x)=0\right\} \\
& =\left\{\left(x_{1}, x_{2}\right): x_{2}=2 x_{1}, 0 \leqslant x_{1} \leqslant 1\right\} .
\end{aligned}
$$

It can be seen that $\phi_{1}(\bar{x})=1, \phi_{2}(\bar{x})=0$, and $\phi_{3}(\bar{x})=-1 / 2$. We have $f_{1}(x)-\phi_{1}(\bar{x}) g_{1}(x)=\left|x_{1}\right|$. Thus, $\bar{x}$ is a solution of Problem

$$
\min \left\{f_{1}(x)-\phi_{1}(\bar{x}) g_{1}(x): x \in M\right\} .
$$


Hence, $\bar{x}$ is a solution of the problem:

$$
\min \left\{\frac{f_{1}(x)}{g_{1}(x)}: x \in M\right\} .
$$

Therefore, $\bar{x}$ is a weak efficient solution of Problem $\left(P_{3}\right)$. It can be seen that

$$
\begin{aligned}
& \partial^{*} f_{1}(0)=\{(-2,-1),(0,-1)\} \\
& \partial^{*} g_{1}(0)=\{(0,-1)\} \\
& \partial^{*} f_{2}(0)=\{(-1,0),(1,0)\} \\
& \partial^{*} g_{2}(0)=\{(0,-1)\} \\
& \partial^{*} f_{3}(0)=\{(0,0),(1,0\} \\
& \partial^{*} g_{3}(0)=\{(0,-1)\} \\
& \partial^{*} h_{1}(0)=\{(-1,0)\} \\
& \partial^{*} h_{2}(0)=\{(0,0),(1,0)\} \\
& \partial^{*} \ell(0)=\{(-2,1)\}
\end{aligned}
$$

One has $T(D ; \bar{x})=\mathbb{R}_{+}^{2}, N(D ; \bar{x})=\mathbb{R}_{-}^{2}$. The constraint qualification (CQ) is satisfied with $v_{0}=$ $(1,2) \in \mathbb{R}_{+}^{2}=T(D ; \bar{x})$. Thus all the hypotheses of Theorem 4.1 are fulfilled, and Kuhn-Tucker necessary condition (4.1) holds with $\alpha_{1}^{*}=2, \alpha_{2}^{*}=1, \alpha_{3}^{*}=2, \mu_{1}^{*}=2, \mu_{2}^{*}=1, v^{*}=1$

\section{SUFFICIENT OPTIMALITY CONDITIONS}

To derive sufficient optimality conditions, we need some assumptions on the generalized convexity. Adapting to the definitions of asymptotic pseudoconvex and quasiconvex functions in [10], we introduce the following definitions.

Definition 5.1. Let $f: X \rightarrow \mathbb{R}$ be a function which admits a convexificator $\partial^{*} f(x) \subset X^{*}$, and given the mapping $\eta: X \times X \rightarrow X$.

(1) We say that $f$ is asymptotic pseudoinvex at $\bar{x}$ with respect to $(\eta, D)$ if, for every $x \in D$,

$$
\text { for some } x_{n}^{*} \in \operatorname{conv} \partial^{*} f(\bar{x}), \lim _{n \rightarrow \infty}\left\langle x_{n}^{*}, \eta(x, \bar{x})\right\rangle \geqslant 0 \Longrightarrow f(x) \geqslant f(\bar{x}) ;
$$

(2) We say that $f$ is asymptotic strict pseudoinvex at $\bar{x}$ with respect to $(\eta, D)$ if, for every $x \in D, x \neq \bar{x}$,

$$
\text { for some } x_{n}^{*} \in \operatorname{conv} \partial^{*} f(\bar{x}), \lim _{n \rightarrow \infty}\left\langle x_{n}^{*}, \eta(x, \bar{x})\right\rangle \geqslant 0 \Longrightarrow f(x)>f(\bar{x}) ;
$$

(3) The function $f$ is called asymptotic quasiinvex at $\bar{x}$ with respect to $(\eta, D)$ if, for all $x \in D$,

$$
f(x) \leqslant f(\bar{x}) \Longrightarrow \text { for any } x_{n}^{*} \in \operatorname{conv} \partial^{*} f(\bar{x}), \lim _{n \rightarrow \infty}\left\langle x_{n}^{*}, \eta(x, \bar{x})\right\rangle \leqslant 0 .
$$

Remark 5.2. For $\eta(x, \bar{x})=x-\bar{x}$, an asymptotyic pseudoinvex or asymptyotic quasiinvex function $f$ becomes asymptotic pseudoconvex or quasiconvex, respectively, at $\bar{x}$ with respect to $C$ as in [10].

We now give a sufficient condition for weak efficiency of (MFP).

Theorem 5.3. Let $\bar{x} \in M$. Assume that, for $i=1, \ldots, p, f_{i}, g_{i}$ admits bounded convexificators $\partial^{*} f_{i}(\bar{x}), \partial^{*} g_{i}(\bar{x})$ at $\bar{x}$, the mappings $\partial^{*} f_{i}, \partial^{*} g_{i}$ are upper semicontinuous at $\bar{x}$. Suppose also that 
(i) there exists vector $\left(\alpha_{1}^{*}, \ldots, \alpha_{p}^{*}\right) \in \mathbb{R}_{+}^{p} \backslash\{0\},\left(\mu_{1}^{*}, \ldots, \mu_{m}^{*}\right) \in \mathbb{R}_{+}^{m+1}$, and $\left(v_{1}^{*}, \ldots, v_{r}^{*}\right) \in \mathbb{R}^{r}$ such that

$$
\begin{aligned}
0 \in \operatorname{cl}\left(\sum_{i=1}^{p} \alpha_{i}^{*}\right. & \left(\operatorname{conv} \partial^{*} f_{i}(\bar{x})-\phi_{i}(\bar{x}) \operatorname{conv} \partial^{*} g_{i}(\bar{x})\right) \\
& \left.+\sum_{j=1}^{m} \mu_{j}^{*} \operatorname{conv} \partial^{*} h_{j}(\bar{x})+\sum_{k=1}^{r} v_{k}^{*} \operatorname{conv} \partial^{*} \ell_{k}(\bar{x})+N(D ; \bar{x})\right)
\end{aligned}
$$

(ii) there exists a function $\eta: X \times X \rightarrow X$ such that $\eta(x, \bar{x}) \in T(D ; \bar{x})$ for any $x \in D$, and the function $\sum_{i=1}^{p} \alpha_{i}^{*}\left(f_{i}-\phi(\bar{x}) g_{i}\right)$ is asymptotic pseudoinvex with respect to $(\eta, D)$ at $\bar{x}$; the function $h_{j}$ are asymptotic quasiinvex at $\bar{x}$ with respect to $(\eta, D)$ for all $j=1, \ldots, m$; the functions $\pm \ell_{k}$ are asymptotic quasiinvex at $\bar{x}$ with respect to $(\eta, D)$ for all $k=1, \ldots, r$.

Then $\bar{x}$ is a weakly efficient solution of (MFP).

Proof. By contradiction, we suppose that $\bar{x}$ is not a weakly efficient solution of (MFP). By an argument analogous to that used for the proof of Theorem 3.3, we deduce that $\bar{x}$ is not a weakly efficient solution of $\left(P_{1}\right)$. Then, there exists a sequence $x_{n} \rightarrow \bar{x}$ such that

$$
\left\{\begin{array}{l}
\left(\varphi_{1}\left(x_{n}\right)-\varphi_{1}(\bar{x}), \ldots, \varphi_{p}\left(x_{n}\right)-\varphi_{p}(\bar{x})\right) \in-\operatorname{int} \mathbb{R}_{+}^{p}, \\
h_{j}\left(x_{n}\right) \leqslant 0 \text { for all } j=1, \ldots, m, \\
\ell_{k}\left(x_{n}\right)=0 \text { for all } k=1, \ldots, r, \\
x_{n} \in D .
\end{array}\right.
$$

Next, let us show that $\sum_{i=1}^{p} \alpha_{i}\left(\partial^{*} f_{i}(\bar{x})-\phi_{i}(\bar{x}) \partial^{*} g_{i}(\bar{x})\right)$ is a convexificator of the function $\sum_{i=1}^{p} \alpha_{i}\left(f_{i}-\right.$ $\left.\phi_{i}(\bar{x}) g_{i}\right)$ at $\bar{x}$. In fact, we set

$$
\begin{gathered}
G\left(\left(u_{1}, v_{1}\right), \ldots,\left(u_{p}, v_{p}\right)\right):=\left(\alpha_{1}\left(u_{1}-\phi_{1}(\bar{x}) v_{1}\right), \ldots, \alpha_{p}\left(u_{p}-\phi_{p}(\bar{x}) v_{p}\right)\right), \\
F(x):=\left(\left(f_{1}(x), g_{1}(x)\right), \ldots,\left(f_{p}(x), g_{p}(x)\right)\right) .
\end{gathered}
$$

Then $G$ is Fréchet differentiable at $\bar{x}$, and $\sum_{i=1}^{p} \alpha_{i}\left(f_{i}-\phi_{i}(\bar{x}) g_{i}\right)(x)=(G \circ F)(x)$. Moreover,

$$
\left.\partial^{*} G(F(\bar{x}))=\{\nabla G(F(\bar{x}))\}=\left\{\left(\alpha_{1}\left(1,-\phi_{1}(\bar{x})\right), \ldots, \alpha_{p}\left(1,-\phi_{p}(\bar{x})\right)\right)\right)\right\} .
$$

Taking account of Proposition 2.2, it follows that the following set is a convexificator of $G \circ F$ at $\bar{x}$

$$
\begin{gathered}
\left(\alpha_{1}\left(1,-\phi_{1}(\bar{x})\right), \ldots, \alpha_{p}\left(1,-\phi_{p}(\bar{x})\right)\right)\left(\left(\partial^{*} f_{1}(\bar{x}), \partial^{*} g_{1}(\bar{x})\right), \ldots,\left(\partial^{*} f_{p}(\bar{x}), \partial^{*} g_{p}(\bar{x})\right)\right) \\
=\sum_{i=1}^{p} \alpha_{i}\left(\partial^{*} f_{i}(\bar{x})-\phi_{i}(\bar{x}) \partial^{*} g_{i}(\bar{x})\right) .
\end{gathered}
$$

It follows from the Kuhn-Tucker conditon (5.1) that there exist $a_{i}^{(n)} \in \partial^{*} \varphi_{i}(\bar{x}), b_{j}^{(n)} \in \partial^{*} h_{j}(\bar{x})$, $c_{k}^{(n)} \in \partial^{*} \ell_{k}(\bar{x}), d^{(n)} \in N(D ; \bar{x})$ such that

$$
\lim _{n \rightarrow \infty}\left(\sum_{i=1}^{p} \alpha_{i}^{*} a_{i}^{(n)}+\sum_{j \in I(\bar{x})} \mu_{j}^{*} b_{j}^{(n)}+\sum_{k=1}^{r} \beta_{k}^{*} c_{k}^{(n)}+d^{(n)}\right)=0 .
$$


Hence,

$$
\begin{gathered}
\lim _{n \rightarrow \infty}\left(\sum_{i=1}^{p} \alpha_{i}^{*}\left\langle a_{i}^{(n)}, \eta\left(x_{n}, \bar{x}\right)\right\rangle+\sum_{j \in I(\bar{x})} \mu_{j}^{*}\left\langle b_{j}^{(n)}, \eta\left(x_{n}, \bar{x}\right)\right\rangle+\sum_{k=1}^{r} \beta_{k}^{*}\left\langle c_{k}^{(n)}, \eta\left(x_{n}, \bar{x}\right)\right\rangle+\left\langle d^{(n)}, \eta\left(x_{n}, \bar{x}\right)\right\rangle\right) \\
=0 .
\end{gathered}
$$

For $x_{n} \in M$, one has $h_{j}\left(x_{n}\right) \leqslant 0=h_{j}(\bar{x})(\forall j \in I(\bar{x}))$. By virtue of the asymptotic quasiinvexity of $h_{j}$, it follows that

$$
\lim _{n \rightarrow \infty}\left\langle b_{j}^{(n)}, \eta\left(x_{n}, \bar{x}\right)\right\rangle \leqslant 0 .
$$

For each $k=1, \ldots, r$, we have $\ell_{k}\left(x_{n}\right)=0=\ell_{k}(\bar{x})(\forall k \in L)$. In view of the asymptotic quasiinvexity of $\pm v_{k}^{*} \ell_{k}$, it results that

$$
\lim _{n \rightarrow \infty}\left\langle c_{k}^{(n)}, \eta\left(x_{n}, \bar{x}\right)\right\rangle=0
$$

Since $x_{n} \in D$ and $d^{(n)} \in N(D ; \bar{x})$, it holds that

$$
\lim _{n \rightarrow \infty}\left\langle d^{(n)}, \eta\left(x_{n}, \bar{x}\right)\right\rangle \leqslant 0 .
$$

Combining (5.2)-(5.5) yields that

$$
\lim _{n \rightarrow \infty}\left\langle\sum_{i=1}^{p} \alpha_{i}^{*} a_{i}^{(n)}, \eta\left(x_{n}, \bar{x}\right)\right\rangle \geqslant 0 .
$$

By the asymptotic pseudoinvexity of $\sum_{i=1}^{p} \alpha_{i}^{*}\left(f_{i}-\phi_{i}(\bar{x}) g_{i}\right)$, it follows that, for all $x \in M$,

$$
\sum_{i=1}^{p} \alpha_{i}^{*}\left(f_{i}(x)-\phi_{i}(\bar{x}) g_{i}(x)\right) \geqslant \sum_{i=1}^{p} \alpha_{i}^{*}\left(f_{i}(\bar{x})-\phi_{i}(\bar{x}) g_{i}(\bar{x})\right)=0
$$

Hence, $\bar{x}$ is a weakly efficient solution of (MFP). If not, there exists $x_{1} \in M$ such that

$$
\frac{f_{i}\left(x_{1}\right)}{g_{i}\left(x_{1}\right)}-\frac{f_{i}(\bar{x})}{g_{i}(\bar{x})}<0 \quad i=1, \ldots, p .
$$

The latter implies that

$$
f_{i}\left(x_{1}\right)-\phi_{i}(\bar{x}) g_{i}\left(x_{1}\right)<0, \quad i=1, \ldots, p .
$$

Since $\left(\alpha_{1}^{*}, \ldots, \alpha_{p}^{*}\right) \neq 0$, it follows from (5.8) that

$$
\sum_{i=1}^{p} \alpha_{i}^{*}\left(f_{i}\left(x_{1}\right)-\phi_{i}(\bar{x}) g_{i}\left(x_{1}\right)\right)<0 .
$$

But this conflicts with (5.7). The proof is complete.

\section{Duality}

We now consider the following dual problem of Mond-Weir type for Problem (MFP): 


$$
\begin{aligned}
& \max \phi(u):=\left(\frac{f_{1}(u)}{g_{1}(u)}, \ldots, \frac{f_{p}(u)}{g_{p}(u)}\right), \\
(M W D P) \quad \text { s.t. } \quad & 0 \in \operatorname{cl}\left(\sum_{i=1}^{p} \alpha_{i}\left(\operatorname{conv} \partial^{*} f_{i}(u)-\phi_{i}(u) \operatorname{conv} \partial^{*} g_{i}(u)\right)\right. \\
& \left.+\sum_{j=1}^{m} \mu_{j} \operatorname{conv} \partial^{*} h_{j}(u)+\sum_{k=1}^{r} v_{k} \operatorname{conv} \partial^{*} \ell_{k}(u)+N(D ; u)\right), \\
& \mu_{j} h_{j}(u) \geqslant 0(i \in I(u)), v_{k} \ell_{k}(u)=0(k=1, \ldots, L), u \in D, \\
& \left(\alpha_{1}, \ldots, \alpha_{p}\right) \in \mathbb{R}_{+}^{p} \backslash\{0\}, \mu_{i} \geqslant 0(i \in I(u)), \mu_{r}=0(r \notin I(u)), \\
& v_{k} \in \mathbb{R}(k \in L) .
\end{aligned}
$$

Denote by $M_{1}$ the feasible set of Problem (MWDP). We set $\lambda:=\left(\lambda_{1}, \ldots, \lambda_{p}\right), \mu:=\left(\mu_{i}\right)_{i \in I}$, $v:=\left(v_{k}\right)_{k \in L}$. Recall that $\bar{x}$ is called a local weak efficient maximum of (MWDP) if there exists a number $\delta>0$ such that, for all $x \in M_{1} \cap B(\bar{x}, \delta)$,

$$
\phi(x)-\phi(\bar{x}) \notin \operatorname{int} Q .
$$

A weak duality theorem for the primal problem (FMP) and the dual problem (MWDP) can be stated as follows.

Theorem 6.1. (Weak duality) Let $x$ and $(u, \alpha, \mu, v)$ be the feasible points of (MFP) and (MWDP), respectively. Assume that

(i) $f_{i}, g_{i}, \ell_{k}$ are continuous and admit convexificators $\partial^{*} f_{i}(y), \partial^{*} g_{i}(y)$, at y near $u$, respectively. They admit bounded convexificators $\partial^{*} f_{i}(u), \partial^{*} g_{i}(u)$ at $u$. Moreover, $\partial^{*} f_{i}, \partial^{*} g_{i}$ are upper semicontinuous at $u$;

(ii) there exists a function $\eta: X \times X \rightarrow X$ such that $\eta(y, u) \in T(D ; u)$ for any $y \in D$, and the function $\sum_{i=1}^{p} \alpha_{i}^{*}\left(f_{i}-\phi(u) g_{i}\right.$ is asymptotic pseudoinvex with respect to $(\eta, D)$ at $u$; the function $\mu_{j} h_{j}$ are asymptotic quasiinvex at $\bar{x}$ with respect to $(\eta, D)$ for all $j=1, \ldots, m$; the functions $\pm v_{l} \ell_{k}$ are asymptotic quasiinvex at $u$ with respect to $(\eta, D)$ for all $k=$ $1, \ldots, r$.

Then

$$
\phi(x) \nless \phi(u) .
$$

Proof. Due to $(u, \lambda, \mu, \gamma) \in M_{1}$, there exist $\xi_{i}^{(n)} \in \operatorname{conv} \partial^{*} f_{i}(u)(i=1, \ldots, p), \theta_{i}^{(n)} \in \operatorname{conv} \partial^{*} g_{i}(u)$ $(i=1, \ldots, p), \zeta_{j}^{(n)} \in \operatorname{conv} \partial^{*}\left(\mu_{j} h_{j}\right)(u)(j \in L), \chi_{k}^{(n)} \in \operatorname{conv} \partial^{*}\left(v_{k} \ell_{k}\right)(u)(k \in L), \sigma \in N(D, u)$ such that

$$
0=\lim _{n \rightarrow \infty}\left[\sum_{i=1}^{p} \alpha_{i}\left(\xi_{i}^{(n)}-\phi_{i}(u) \theta_{i}^{(n)}\right)+\sum_{j \in I(u)} \zeta_{j}^{(n)}+\sum_{k \in L} \chi_{k}^{(n)}+\sigma^{(n)}\right] .
$$

Hence, for $x \in M$, we have

$$
\begin{aligned}
\lim _{n \rightarrow \infty}\left\langle\lambda _ { i } \sum _ { i = 1 } ^ { p } \alpha _ { i } \left(\xi_{i}^{(n)}\right.\right. & \left.\left.-\phi_{i}(u) \theta_{i}^{(n)}\right), \eta(x, u)\right\rangle+\lim _{n \rightarrow \infty} \sum_{j \in I(u)}\left\langle\zeta_{j}^{(n)}, \eta(x, u)\right\rangle \\
& +\sum_{k \in L} \lim _{n \rightarrow \infty}\left\langle\chi_{k}^{(n)}, \eta(x, u)\right\rangle+\lim _{n \rightarrow \infty}\left\langle\sigma^{(n)}, \eta(x, u)\right\rangle=0 .
\end{aligned}
$$


Observe that, for $x \in M, \mu_{j} h_{j}(x) \leqslant 0 \leqslant \mu_{j} h_{j}(u)(\forall j \in I(u))$. In view of the asymptotic quasiconvexity of $\mu_{j} h_{j}$ at $u(i \in I(u))$, it results that

$$
\lim _{n \rightarrow \infty}\left\langle\zeta_{j}^{(n)}, \eta(x, u)\right\rangle \leqslant 0 .
$$

Since $\ell_{k}(x)=0=\ell_{k}(u)$, by virtue of the asymptotic quasiinvexity of $\pm v_{k} h_{k}(\forall k \in L)$, for $x \in M$, we have

$$
\lim _{n \rightarrow \infty}\left\langle\chi_{k}^{(n)}, \eta(x, u)\right\rangle=0 .
$$

The convexity of $D$ implies that, for $x \in M$,

$$
\lim _{n \rightarrow \infty}\left\langle\sigma^{(n)}, \eta(x, u)\right\rangle \leqslant 0 .
$$

Let us show that $\partial^{*} f_{i}(u)-\phi_{i}(u) \partial^{*} g_{i}(u)$ is a convexificator of the function $\varphi_{i}:=f_{i}-\phi_{i}(u) g_{i}$ at $u(i=1, \ldots, p)$. In fact, for $i=1, \ldots, p$, we set

$$
\begin{gathered}
G\left(v_{i}, w_{i}\right):=v_{i}-\phi_{i}(u) w_{i}, \\
F(x):=\left(f_{i}(x), g_{i}(x)\right) .
\end{gathered}
$$

Then, $G$ is Fréchet differentiable at $u$, and

$$
\left.\partial^{*} G(u)\right)=\{\nabla G(u)\}=\left\{\left(1,-\phi_{i}(u)\right)\right\} .
$$

Taking account of Proposition 2.2, it follows that the following set is a convexificator of $\varphi_{i}:=$ $G \circ F$ at $u$

$$
\left.\left(1,-\phi_{i}(u)\right)\right)\left(\left(\partial^{*} f_{i}(u), \partial^{*} g_{i}(u)\right)\right)=\partial^{*} f_{i}(u)-\phi_{i}(u) \partial^{*} g_{i}(u) .
$$

Combining (6.1)-(6.4) yields that

$$
\lim _{n \rightarrow \infty} \sum_{i=1}^{p}\left\langle\alpha_{i}\left(\xi_{i}^{(n)}-\phi_{i}(u) \eta_{i}^{(n)}, \eta(x, u)\right\rangle \geqslant 0 .\right.
$$

Since the function $\sum_{i=1}^{p} \alpha_{i}^{*}\left(f_{i}-\phi_{i}(u) g_{i}\right)$ is asymptotic pseudoinvex with respect to $(\eta, D)$ at $u$, it follows that, for every $x \in M$,

$$
\sum_{i=1}^{p} \alpha_{i}\left(f_{i}(x)-\phi_{i}(u) g_{i}(x)\right) \geqslant \sum_{i=1}^{p} \alpha_{i}\left(f_{i}(u)-\phi_{i}(u) g_{i}(u)\right)=0 .
$$

Hence,

$$
\phi(x) \nless \phi(u) .
$$

In fact, if not, there exists $x_{1} \in M$ such that

$$
\phi_{i}\left(x_{1}\right)<\phi_{i}(u)(i=1, \ldots, p),
$$

which implies that

$$
\frac{f_{i}\left(x_{1}\right)}{g_{i}\left(x_{1}\right)}-\phi_{i}(u)<0(i=1, \ldots, p)
$$

Hence,

$$
\left(f_{i}\left(x_{1}\right)-\phi_{i}(u) g_{i}\left(x_{1}\right)\right)<0=\left(f_{i}(u)-\phi_{i}(u) g_{i}(u)\right),(\forall i=1, \ldots, p) .
$$

Since $\left(\alpha_{1}^{*}, \ldots, \alpha_{p}^{*}\right) \in \mathbb{R}_{+}^{p} \backslash\{0\}$, we have

$$
\sum_{i=1}^{p} \alpha_{i}\left(f_{i}\left(x_{1}\right)-\phi_{i}(u) g_{i}\left(x_{1}\right)\right)<\sum_{i=1}^{p} \alpha_{i}\left(f_{i}(u)-\phi_{i}(u) g_{i}(u) .\right.
$$


So, we reach a contradiction to (6.5). Hence, (6.6) holds and the proof is complete.

In what follows, we give a strong duality theorem for (MFP) and (MWDP).

Theorem 6.2. (Strong duality) Let $\bar{x}$ be a local weak efficient solution of (MFP). Assume that all the hypotheses of Theorem 4.1 are fulfilled. Then there exist $\bar{\alpha}_{i} \geqslant 0(s=1, \ldots, p)$, not all zero, $\bar{\mu}_{j} \geqslant 0(\forall j \in I(\bar{x})), \bar{v}_{k} \in \mathbb{R}(\forall k \in L)$ such that $(\bar{x}, \bar{\lambda}, \bar{\mu}, \bar{v})$ is a feasible point of (MWDP), and the value of the objective functions of (MFP) and (MWDP) at $\bar{x}$ and $(\bar{x}, \bar{\lambda}, \bar{\mu}, \bar{\gamma})$, respectively, are equal. Moreover, if the Assumption (ii) of Theorem 6.1 holds, then $(\bar{x}, \bar{\lambda}, \bar{\mu}, \bar{v})$ is a weakly efficient solution of (MWDP).

Proof. Since $\bar{x}$ is a local weak solution of (MFP), we can invoke Theorem 4.1 to deduce that there exist $\bar{\alpha}_{i} \geqslant 0(i=1,, \ldots, p)$, not all zero, $\bar{\mu}_{j} \geqslant 0(\forall i \in I(\bar{x})), \bar{v}_{k} \in \mathbb{R}(\forall k \in L)$ such that

$$
\begin{aligned}
0 \in \operatorname{cl} & \left(\sum_{i=1}^{p} \bar{\alpha}_{i}\left(\operatorname{conv} \partial^{*} f(\bar{x})-\phi(\bar{x}) \partial^{*} g_{i}(\bar{x})\right)\right. \\
& \left.+\sum_{i \in I(\bar{x})} \bar{\mu}_{j} \operatorname{conv} \partial^{*} h j(\bar{x})+\sum_{k \in L} \bar{v}_{k} \operatorname{conv} \partial^{*} \ell_{k}(\bar{x})+N(D ; \bar{x})\right) .
\end{aligned}
$$

If $i \notin I(\bar{x})$, we take $\bar{\mu}_{i}=0$. Then, $(\bar{x}, \bar{\lambda}, \bar{\mu}, \bar{\gamma})$ is a feasible point of (MWDP), and the value of the objective functions of (MFP) and (MWDP) at $\bar{x}$ and $(\bar{x}, \bar{\lambda}, \bar{\mu}, \bar{\gamma})$, respectively, are equal. If the Assumption (ii) of Theorem 6.1 holds, then the hypotheses of Theorem 6.1 on weak duality are fulfilled. By this theorem, one gets that, for every feasible points $(u, \lambda, \mu, v)$ of (MWDP),

$$
F(\bar{x}) \nless F(u) .
$$

Hence, $(\bar{x}, \bar{\lambda}, \bar{\mu}, \bar{\gamma})$ is a weakly efficient solution of (MWDP).

Theorem 6.3. (Converse duality) Let $\bar{x}$ and $(\bar{u}, \bar{\alpha}, \bar{\mu}, \bar{v})$ be the feasible points of (MFP) and $(M W D P)$, respectively. Assume that

(i) $f_{i}, g_{i}, \ell_{k}$ are continuous and admit convexificators $\partial^{*} f_{i}(u), \partial^{*} g_{i}(u)$ at u near $\bar{u}$, respectively. They admit bounded convexificators $\partial^{*} f_{i}(\bar{u}), \partial^{*} g_{i}(\bar{u})$ at $\bar{u}$. Moreover, $\partial^{*} f_{i}, \partial^{*} g_{i}$, $\partial_{j}^{*}$, and $\partial^{*} \ell_{k}$ are upper semicontinuous at $\bar{u}$;

(ii) there exists a function $\eta: X \times X \rightarrow X$ such that $\eta(x, \bar{u}) \in T(D ; \bar{u})$ for any $x \in D$, and the function $\sum_{i=1}^{p} \alpha_{i}^{*}\left(f_{i}-\phi(\bar{u}) g_{i}\right)$ is asymptotic strict pseudoinvex with respect to $(\eta, D)$ at $\bar{u}$; $D$ is convex, the function $\bar{\mu}_{j} h_{j}$ are asymptotic quasiinvex at $\bar{u}$ with respect to $(\eta, D)$ for all $j=1, \ldots, m$; the function $\pm \bar{v}_{k} \ell_{k}$ are asymptotic quasiinvex at $\bar{u}$ with respect to $(\eta, D)$ for all $k=1, \ldots, r$. Suppose, furthermore, that

$$
\sum_{i=1}^{p} \bar{\alpha}_{i}\left(f_{i}(\bar{x})-\phi_{i}(\bar{u}) g_{i}(\bar{x})\right) \leqslant \sum_{i=1}^{p} \bar{\alpha}_{i}\left(f_{i}(\bar{u})-\phi_{i}(\bar{u}) g_{i}(\bar{u})\right) .
$$

Then $\bar{u}=\bar{x}$.

Proof. Assume $\bar{u} \neq \bar{x}$. Since $(\bar{u}, \bar{\alpha}, \bar{\mu}, \bar{v})$ is the feasible points of (MWDP), it follows that there exist $\xi_{i}^{(n)} \in \operatorname{conv} \partial^{*} f_{i}(\bar{u})(i=1, \ldots, p), \theta_{i}^{(n)} \in \operatorname{conv} \partial^{*} g_{i}(\bar{u})(i=1, \ldots, p), \zeta_{j}^{(n)} \in \operatorname{conv} \partial^{*}\left(\bar{\mu}_{j} h_{j}\right)(\bar{u})$ $(j \in L), \chi_{k}^{(n)} \in \partial^{*}\left(\bar{v}_{k} \ell_{k}\right)(\bar{u})(k \in L), \sigma^{(n)} \in N(D, \bar{u})$ such that

$$
0=\lim _{n \rightarrow \infty}\left[\sum_{i=1}^{p} \bar{\alpha}_{i}\left(\xi_{i}^{(n)}-\phi_{i}(u) \theta_{i}^{(n)}\right)+\sum_{j \in I(\bar{u})} \zeta_{j}^{(n)}+\sum_{k \in L} \chi_{k}^{(n)}+\sigma^{(n)}\right] .
$$


Observe that, for $x \in M, \bar{\mu}_{j} h_{j}(x) \leqslant 0 \leqslant \bar{\mu}_{j} h_{j}(u)(\forall j \in I(\bar{u}))$. In view of the asymptotic quasiconvexity of $\bar{\mu}_{j} h_{j}$ at $\bar{u}(i \in I(\bar{u}))$, we have

$$
\lim _{n \rightarrow \infty}\left\langle\zeta_{j}^{(n)}, \eta(x, \bar{u})\right\rangle \leqslant 0 .
$$

Since $\ell_{k}(x)=0=\ell_{k}(u)$, in view of the asymptotic quasiinvex of $\pm \bar{v}_{k} h_{k}(\forall k \in L)$, for $x \in M$, we have

$$
\lim _{n \rightarrow \infty}\left\langle\chi_{k}^{(n)}, \eta(x, \bar{u})\right\rangle=0 .
$$

The convexity of $D$ implies that, for $x \in M$,

$$
\lim _{n \rightarrow \infty}\left\langle\sigma^{(n)}, \eta(x, \bar{u})\right\rangle \leqslant 0 .
$$

Let us show that $\partial^{*} f_{i}(\bar{u})-\phi_{i}(\bar{u}) \partial^{*} g_{i}(\bar{u})$, which is a convexificator of the function $\varphi_{i}:=f_{i}-$ $\phi_{i}(\bar{u}) g_{i}$ at $\bar{u}(i=1, \ldots, p)$. In fact, for $i=1, \ldots, p$, we set

$$
\begin{gathered}
G\left(v_{i}, w_{i}\right):=v_{i}-\phi_{i}(\bar{u}) w_{i}, \\
F(x):=\left(f_{i}(x), g_{i}(x)\right) .
\end{gathered}
$$

Then, $G$ is Fréchet differentiable at $\bar{u}$, and

$$
\left.\partial^{*} G(\bar{u})\right)=\{\nabla G(\bar{u})\}=\left\{\left(1,-\phi_{i}(\bar{u})\right)\right\} .
$$

Taking account of Proposition 2.2, it follows that the following set is a convexificator of $\varphi_{i}:=$ $G \circ F$ at $\bar{u}$

$$
\left(1,-\phi_{i}(\bar{u})\right)\left(\partial^{*} f_{i}(\bar{u}), \partial^{*} g_{i}(\bar{u})\right)=\partial^{*} f_{i}(\bar{u})-\phi_{i}(\bar{u}) \partial^{*} g_{i}(\bar{u}) .
$$

Combining (6.8)-(6.11) yields that

$$
\lim _{n \rightarrow \infty} \sum_{i=1}^{p}\left\langle\bar{\alpha}_{i}\left(\xi_{i}^{(n)}-\phi_{i}(\bar{u}) \theta_{i}^{(n)}\right), \eta(x, \bar{u})\right\rangle \geqslant 0 .
$$

Since $\left.\sum_{i=1}^{p} \bar{\alpha}_{i}\left(f_{i}-\phi_{i}(\bar{u}) g_{i}\right)\right)$ is asymptotic strict pseudoinvex with respect to $(\eta, D)$ at $\bar{u}$, it follows that

$$
\left.\sum_{i=1}^{p} \bar{\alpha}_{i}\left(f_{i} \bar{x}\right)-\phi_{i}(\bar{u}) g_{i}(\bar{x})\right)>\sum_{i=1}^{p} \bar{\alpha}_{i}\left(f_{i}(\bar{u})-\phi_{i}(\bar{u}) g_{i}(\bar{u})\right) .
$$

But this contradicts (6.7). Hence, $\bar{u}=\bar{x}$.

Now we introduce the following dual problem of Wolfe type for (MFP):

$$
\max \phi(x)+\sum_{j \in I(u)} \mu_{j} h_{j}(u)+\sum_{k \in L} v_{k} \ell_{k}(u)
$$

(WDP) s.t. $\quad 0 \in \operatorname{cl}\left(\sum_{i=1}^{p} \alpha_{i}\left(\operatorname{conv} \partial^{*} f_{i}(u)-\phi_{i}(u) \operatorname{conv} \partial^{*} g_{i}(u)\right)\right.$

$$
\begin{aligned}
& \left.+\sum_{j \in I(u)} \mu_{i} \operatorname{conv} \partial^{*} h_{j}(u)+\sum_{k \in L} v_{k} \operatorname{conv} \partial^{*} \ell_{k}(u)+N(D, u)\right), \\
& u \in D, \alpha \in \mathbb{R}_{+}^{m} \backslash\{0\}, \sum_{i=1}^{m} \alpha_{i}=1, \\
& \mu_{j} h_{j}(u)=0(i \in I), \ell_{k}(u)=0, v_{k} \in \mathbb{R}(k \in L) .
\end{aligned}
$$

Denote by $M_{2}$ the feasible set of (WDP). Note that, for $b \in \mathbb{R}, \phi(u)+b:=\left(\phi_{1}(u)+b, \ldots, \phi_{p}(u)+b\right)$. 
A weak duality theorem for (MFP) and (WDP) can be stated as follows.

Theorem 6.4. (Weak duality) Let $x$ and $(u, \alpha, \mu, \gamma)$ be the feasible points of (MFP) and (WDP), respectively. Assume that

(i) $f_{i}, g_{i}, h_{j}, \ell_{k}$ are continuous and admit convexificators $\partial^{*} f_{i}(y), \partial^{*} g_{i}(y), \partial^{*} h_{j}(y), \partial^{*} \ell_{k}(y)$ at $y$ near $x$, respectively. They admit bounded convexificators $\partial^{*} f_{i}(x), \partial^{*} g_{i}(x), \partial^{*} h_{j}(x)$, $\partial^{*} \ell_{k}(x)$ at $x$. Moreover, $\partial^{*} f_{i}, \partial^{*} g_{i}, \partial^{*} h_{j}$, and $\partial^{*} \ell_{k}$ are upper semicontinuous at $\bar{x}$;

(ii) there exists a function $\eta: X \times X \rightarrow X$ such that $\eta(y, u) \in T(D ; u)$ for any $y \in D$, and the function $\sum_{i=1}^{p} \alpha_{i}\left(f_{i}-\phi(\bar{x}) g_{i}\right)$ is asymptotic pseudoinvex with respect to $(\eta, D)$ at $u$.

Then

$$
\phi(x) \nless \phi(u)+\sum_{j \in I(u)} \mu_{j} h_{j}(u)+\sum_{k \in L} v_{k} \ell_{k}(u) .
$$

Proof. Since $(u, \alpha, \mu, \gamma) \in M_{2}$, there exist $\xi_{i}^{(n)} \in \operatorname{conv} \partial^{*} f_{i}(u)(k=1, \ldots, p), \theta_{i}^{(n)} \in \operatorname{conv} \partial^{*} g_{i}(u)$ $(i \in I(u)), \zeta_{j}^{(n)} \in \partial^{*} h_{j}(u), \chi_{k}^{(n)} \in \partial^{*} \ell_{k}(u), \sigma^{(n)} \in N(D, u)$ such that

$$
0=\lim _{n \rightarrow \infty}\left[\alpha_{i}\left(\xi_{i}^{(n)}-\phi_{i}(u) \theta_{i}^{(n)}\right)+\sum_{i \in I(u)} \mu_{j} \zeta_{j}^{(n)}+\sum_{j \in L} v_{k} \chi_{k}^{(n)}+\sigma^{(n)}\right]
$$

Hence, for $x \in M$,

$$
\begin{aligned}
\lim _{n \rightarrow \infty}\left[\left\langle\alpha _ { i } \left(\xi_{i}^{(n)}\right.\right.\right. & \left.\left.-\phi_{i}(u) \theta_{i}^{(n)}\right), \eta(x, u)\right\rangle+\sum_{i \in I(u)} \mu_{j}\left\langle\zeta_{j}^{(n)}, \eta(x, u)\right\rangle \\
& \left.+\sum_{j \in L} v_{k}\left\langle\chi_{k}^{(n)}, \eta(x, u)\right\rangle\right]+\lim _{n \rightarrow \infty}\left\langle\sigma^{(n)}, \eta(x, u)\right\rangle=0 .
\end{aligned}
$$

Since $D$ is convex, for $x \in M$, we have

$$
\lim _{n \rightarrow \infty}\left\langle\sigma^{(n)}, \eta(x, u)\right\rangle \leqslant 0 .
$$

Combining (6.12) and (6.13) yields that

$$
\begin{gathered}
\lim _{n \rightarrow \infty}\left[\left\langle\alpha_{i}\left(\xi_{i}^{(n)}-\phi_{i}(u) \theta_{i}^{(n)}\right), \eta(x, u)\right\rangle+\sum_{i \in I(u)} \mu_{i}\left\langle\zeta_{j}^{(n)}, \eta(x, u)\right\rangle\right. \\
\left.+\sum_{j \in L} v_{k}\left\langle\chi_{k}^{(n)}, \eta(x, u)\right\rangle\right] \geqslant 0 .
\end{gathered}
$$

Let us show that the set

$$
\operatorname{cl}\left(\sum_{i=1}^{p} \alpha_{i}\left(\partial^{*} f_{i}(u)-\phi_{i}(u) \partial^{*} g_{i}(u)\right)+\sum_{j \in I(u)} \mu_{j} \partial^{*} h_{j}(u)+\sum_{k \in L} v_{k} \partial^{*} \ell_{k}(u)\right)
$$

is a convexificator of the following function at $u$ :

$$
\sum_{i=1}^{p} \alpha_{i}\left(f_{i}-\phi_{i}(u) g_{i}\right)+\sum_{j \in I(u)} \mu_{j} h_{j}+\sum_{k \in L} v_{k} \ell_{k}
$$

In fact, we set

$$
G\left(x_{1}, \ldots, x_{p}, y_{1}, \ldots, y_{p}, z_{1}, \ldots, z_{|I(u)|}, w_{1}, \ldots, w_{r}\right):=\sum_{i=1}^{m}\left(x_{i}-\phi_{1}(u) y_{i}\right)+\sum_{j \in I(\bar{u})} \mu_{j} z_{j}+\sum_{k \in L} v_{k} w_{k}
$$




$$
\left.\left.\left.F(x):=\left(f_{1}(x), \ldots, f_{p}(x), g_{1}(x), \ldots, g_{p} x\right), h_{1} x\right), \ldots, h_{|I(u)|}\right)(x), \ell_{1}(x), \ldots, \ell_{r}(x)\right),
$$

where $|I(u)|$ indicates the capacity of $I(u)$. Then

$$
f_{i}(x)-\phi_{i}(x) g_{i}(x)+\sum_{j \in I(u)} \mu_{j} h_{j}(x)+\sum_{k \in L} v_{k} \ell_{k}(x)=(G \circ F)(x) .
$$

It can be seen that $G$ is Fréchet differentiable at $u$. Taking account of Proposition 2.2, it follows that the following set

$$
\left.\partial^{*} f_{i}(u)-\phi_{i}(u) \partial^{*} g_{i}(u)+\sum_{j \in I(u)} \mu_{j} \partial^{*} h_{j}(u)\right)+\sum_{k \in L} v_{k} \partial^{*} \ell_{k}(u)
$$

is a convexificator of the function $\sum_{i=1}^{p} \alpha_{i}\left(f_{i}-\phi_{i}(u) g_{i}\right)+\sum_{j \in I(u)} \mu_{j} h_{j}+\sum_{k \in L} v_{k} \ell_{k}$ at $u$. Consequently,

$$
\begin{aligned}
\sum_{i=1}^{p} \alpha_{i}\left(f_{i}(x)-\phi_{i}(u) g_{i}(x)\right) & +\sum_{j \in I(u)} \mu_{j} h_{j}(x)+\sum_{k \in L} v_{k} \ell_{k}(x) \\
& \geqslant \sum_{i=1}^{p} \alpha_{i}\left(f_{i}(u)+\phi_{i}(u) g_{i}(u)\right)+\sum_{j \in I(u)} \mu_{j} h_{j}(u)+\sum_{k \in L} v_{k} \ell_{k}(u) .
\end{aligned}
$$

Since $h_{j}(x) \leqslant 0(\forall j \in L), \mu_{r}=0(\forall r \notin I(u)), \ell_{k}(x)=0(\forall k \in L)$, it follows from this that

$$
\sum_{i=1}^{p} \alpha_{i}\left(f_{i}(x)-\phi_{i}(x) g_{i}(x)\right) \geqslant \sum_{i=1}^{p} \alpha_{i}\left(f_{i}(u)-\phi_{i}(u) g_{i}(u)\right)+\sum_{j=1}^{m} \mu_{j} h_{j}(u)+\sum_{j=1}^{\ell} v_{k} \ell_{k}(u) .
$$

Then,

$$
\phi(x) \nless \phi(u)+\sum_{j=1}^{\ell} \gamma_{j} h_{j}(u)+\sum_{k \in L} v_{k} \ell_{k}(u) .
$$

Indeed, if not, there exists $x_{1} \in M$ such that

$$
\phi_{i}\left(x_{1}\right)<\phi_{i}(u)+\sum_{i=1}^{m} \mu_{j} h_{j}(u)+\sum_{k \in L} v_{k} \ell_{k}(u)(\forall i=1, \ldots, p) .
$$

Since $x_{1} \in M, u \in M_{2}$, it results that

$$
\begin{aligned}
\phi_{i}\left(x_{1}\right)+\sum_{j=1}^{m} \mu_{j} h_{j}\left(x_{1}\right)+\sum_{k \in L} v_{k} \ell_{k}\left(x_{1}\right) & \leqslant \phi_{i}\left(x_{1}\right) \\
& <\phi_{i}(u)+\sum_{j=1}^{m} \mu_{j} h_{j}(u)+\sum_{k \in L} v_{k} \ell_{k}(u) \\
& =\phi_{i}(u) .
\end{aligned}
$$

Thus

$$
\frac{f_{i}\left(x_{1}\right)}{g_{i}\left(x_{1}\right)}-\phi_{i}(u)<0
$$

and

$$
f_{i}\left(x_{1}\right)-\phi_{i}(u) g_{i}\left(x_{1}\right)<0 .
$$


Since $\alpha_{i} \geqslant 0, \sum_{i=1}^{p} \alpha_{i}=1$, and $f_{i}(u)-\phi_{i}(u) g_{i}(u)=0$, we have

$$
\begin{aligned}
\sum_{i=1}^{p} \alpha_{i}\left(f_{i}\left(x_{1}\right)-\phi_{i}(u) g_{i}\left(x_{1}\right)\right) & <0 \\
& =\sum_{i=1}^{p} \alpha_{i}\left(f_{i}(u)-\phi_{i}(u) g_{i}(u)\right) \\
& =\sum_{i=1}^{p} \alpha_{i}\left(f_{i}(u)-\phi_{i}(u) g_{i}(u)\right)+\sum_{j=1}^{m} \mu_{j} h_{j}(u)+\sum_{j=1}^{\ell} v_{k} \ell_{k}(u) .
\end{aligned}
$$

But this contradicts (6.15). Consequently, (6.16) holds. This completes the proof.

A strong duality theorem for (FMP) and (WDP) can be stated as follows.

Theorem 6.5. (Strong duality) Let $\bar{x} \in M$ be a local weak efficient solution of (MFP), and let all the hypotheses of Theorem 4.1 be fulfilled. Then, there exists vectors $\left(\bar{\alpha}_{1}, \ldots, \bar{\alpha}_{p}\right) \in \mathbb{R}_{+}^{p} \backslash\{0\}$, $\left(\bar{\mu}_{1}, \ldots, \bar{\mu}_{m}\right) \in \mathbb{R}_{+}^{m+1}$, and $\left(\bar{v}_{1}, \ldots, \bar{v}_{r}\right) \in \mathbb{R}^{r}$ such that $(\bar{x}, \bar{\lambda}, \bar{\mu}, \bar{v})$ is a feasible point of (WDP), and the value of the objective functions of (MFP) and (WDP) at $\bar{x}$ and $(\bar{x}, \bar{\lambda}, \bar{\mu}, \bar{v})$, respectively, are equal. Moreover, if assumptions of Theorem 6.4 hold, then $(\bar{x}, \bar{\lambda}, \bar{\mu}, \bar{v})$ is a weak efficient solution of (WDP).

Proof. Since $\bar{x}$ is a local weak efficient solution of (MFP), we invoke Theorem 4.1 to deduce that there exist vectors $\left(\bar{\alpha}_{1}, \ldots, \bar{\alpha}_{p}\right) \in \mathbb{R}_{+}^{p} \backslash\{0\},\left(\bar{\mu}_{1}, \ldots, \bar{\mu}_{m}\right) \in \mathbb{R}_{+}^{m+1}$, and $\left(\bar{v}_{1}, \ldots, \bar{v}_{r}\right) \in \mathbb{R}^{r}$ such that

$$
\begin{aligned}
0 \in \operatorname{cl}\left(\sum_{i=1}^{p} \bar{\alpha}_{i}\left(\operatorname{conv} \partial^{*} f_{i}(u)-\phi_{i}(u) \operatorname{conv} \partial^{*} g_{i}(u)\right)\right. \\
\left.\quad+\sum_{j \in I(u)} \bar{\mu}_{i} \operatorname{conv} \partial^{*} h_{j}(u)+\sum_{k \in L} \bar{v}_{k} \operatorname{conv} \partial^{*} \ell_{k}(u)+N(D, u)\right),
\end{aligned}
$$

by following the proof of Theorem 6.2. Taking $\bar{\mu}_{i}=0$ if $i \notin I(\bar{x})$, we obtain $(\bar{x}, \bar{\lambda}, \bar{\mu}, \bar{v})$ is a feasible point of (WDP), and the value of the objective functions at $\bar{x}$ and $(\bar{x}, \bar{\lambda}, \bar{\mu}, \bar{v}$ ) of (MFP) and (WDP), respectively, are equal.

If all the assumptions of Theorem 6.4 are fulfilled, we deduce that, for every feasible points $(u, \lambda, \mu, \gamma)$ of (WDP),

$$
\begin{aligned}
F(\bar{x})+\sum_{i=1}^{m} \bar{\mu}_{i} g_{i}(\bar{x})+\sum_{j=1}^{\ell} \bar{v}_{k} h_{k}(\bar{x}) & =F(\bar{x}) \\
& \nless F(u)+\sum_{i=1}^{m} \mu_{i} g_{i}(u)+\sum_{j=1}^{\ell} \gamma_{j} h_{j}(u)
\end{aligned}
$$

due to $\bar{x} \in M$. Consequently, $(\bar{x}, \bar{\lambda}, \bar{\mu}, \bar{\gamma})$ is a weak efficient solution of (WDP).

Theorem 6.6. (Converse duality) Let $\bar{x}$ and $(\bar{u}, \bar{\alpha}, \bar{\mu}, \bar{v})$ be the feasible points of (MFP) and (WDP), respectively. Assume that

(i) $f_{i}, g_{i}, h_{j} \ell_{k}$ are continuous and admit convexificators $\partial^{*} f_{i}(u), \partial^{*} g_{i}(u), \partial^{*} h_{j}(u), \partial^{*} \ell_{k}(u)$ at $u$ near $\bar{u}$, respectively. They admit bounded convexificators $\partial^{*} f_{i}(\bar{u}), \partial^{*} g_{i}(\overline{\bar{u}}), \partial^{*} h_{j}(\bar{u})$, $\partial^{*} \ell_{k}(\bar{u})$ at $\bar{u}$. Moreover, $\partial^{*} f_{i}, \partial^{*} g_{i}, \partial^{*} h_{j}$, and $\partial^{*} \ell_{k}$ are upper semicontinuous at $\bar{u}$; 
(ii) there exists a function $\eta: X \times X \rightarrow X$ such that $\eta(x, \bar{u}) \in T(C ; \bar{u})$ for any $x \in C$, and the function $\sum_{i=1}^{p} \alpha_{i}^{*}\left(f_{i}-\phi(u) g_{i}\right)+\sum_{j \in I(\bar{u})} \mu_{j} h_{j}+\sum_{k \in L} v_{k} \ell_{k}$ is strict asymptotic pseudoinvex with respect to $(\eta, D)$ at $u$; $C$ is convex. Suppose, furthermore, that

$$
\begin{aligned}
\sum_{i=1}^{p} \bar{\alpha}_{i}\left(f_{i}(\bar{x})-\phi_{i}(\bar{u}) g_{i}(\bar{x})\right) & +\sum_{j \in I(\bar{u})} \bar{\mu}_{j} h_{j}(\bar{x})+\sum_{k \in L} \bar{v}_{k} \ell_{k}(\bar{x}) \\
& \leqslant \sum_{i=1}^{p} \bar{\alpha}_{i}\left(f_{i}(\bar{u})-\phi_{i}(\bar{u}) g_{i}(\bar{u})\right)+\sum_{j \in I(\bar{u})} \bar{\mu}_{j} h_{j}(\bar{u})+\sum_{k \in L} \bar{v}_{k} \ell_{k}(\bar{u}) .
\end{aligned}
$$

Then $\bar{u}=\bar{x}$.

Proof. Assume $\bar{u} \neq \bar{x}$. Since $(\bar{u}, \bar{\alpha}, \bar{\mu}, \bar{v})$ is the feasible point of (WDP), it follows that there exist $\xi_{i}^{(n)} \in \operatorname{conv} \partial^{*} f_{i}(\bar{u})(i=1, \ldots, p), \theta_{i}^{(n)} \in \operatorname{conv} \partial^{*} g_{i}(\bar{u})(i=1, \ldots, p), \zeta_{j}^{(n)} \in \operatorname{conv} \partial^{*}\left(\bar{\mu}_{j} h_{j}\right)(\bar{u})(j \in$ $L), \chi_{k}^{(n)} \in \operatorname{conv} \partial^{*}\left(\bar{v}_{k} \ell_{k}\right)(\bar{u})(k \in L), \sigma^{(n)} \in N(D, \bar{u})$ such that

$$
0=\lim _{n \rightarrow \infty}\left[\sum_{i=1}^{p} \alpha_{i}\left(\xi_{i}^{(n)}-\phi_{i}(u) \theta_{i}^{(n)}\right)+\sum_{j \in I(u)} \zeta_{j}^{(n)}+\sum_{k \in L} \chi_{k}^{(n)}+\sigma^{(n)}\right] .
$$

The convexity of $D$ implies that, for $x \in M$,

$$
\lim _{n \rightarrow \infty}\left\langle\sigma^{(n)}, \eta(x, \bar{u})\right\rangle \leqslant 0
$$

Let us show that the set

$$
\sum_{i=1}^{p} \bar{\alpha}_{i}\left(\partial^{*} f_{i}(\bar{u})-\phi_{i}(\bar{u}) \partial^{*} g_{i}(\bar{u})\right)+\sum_{j \in I(\bar{u})} \bar{\mu}_{j} \partial^{*} h_{j}(\bar{u})+\sum_{k \in L} \bar{v}_{k} \partial^{*} \ell_{k}(\bar{u})
$$

is a convexificator of the the following function at $\bar{u}$ :

$$
\sum_{i=1}^{p} \bar{\alpha}_{i}\left(f_{i}-\phi_{i}(\bar{u}) g_{i}\right)+\sum_{j \in I(\bar{u})} \bar{\mu}_{j} h_{j}+\sum_{k \in L} \bar{v}_{k} \ell_{k}
$$

In fact, we set

$$
\begin{gathered}
G\left(x_{1}, \ldots, x_{p}, y_{1}, \ldots, y_{p}, z_{1}, \ldots, z_{|I(\bar{u})|}, w_{1}, \ldots, w_{r}\right):=\sum_{i=1}^{m}\left(x_{i}-\phi_{1}(\bar{u}) y_{i}\right)+\sum_{j \in I(\bar{u})} \mu_{j} z_{j}+\sum_{k=1}^{r} v_{k} w_{k}, \\
F(x):=\left(f_{1}(x), \ldots, f_{p}(x), g_{1}(x), \ldots, g_{p}(x), h_{1}(x), \ldots, h_{|I(\bar{u})|}(x), \ell_{1}(x), \ldots, \ell_{r}(x)\right) .
\end{gathered}
$$

Then, $G$ is Fréchet differentiable at $\bar{x}$. Taking account of Proposition 2.2, it follows that the following set: $\left.\sum_{i=1}^{p} \bar{\alpha}_{i}\left(\partial^{*} f_{i}(\bar{u})-\phi_{i}(\bar{u}) \partial^{*} g_{i}(\bar{u})\right)+\sum_{j \in I(\bar{u})} \bar{\mu}_{j} \partial^{*} h_{j}(\bar{u})\right)+\sum_{k \in L} v_{k} \partial^{*} \ell_{k}(\bar{u})$, which is a convexificator of the function $\sum_{i=1}^{p} \bar{\alpha}_{i}\left(f_{i}-\phi_{i}(\bar{x}) g_{i}\right)+\sum_{j \in I(\bar{u})} \bar{\mu}_{j} h_{j}+\sum_{k \in L}$ at $\bar{u}$. Combining (6.18), (6.19) yields that

$$
\lim _{n \rightarrow \infty}\left\langle\bar{\alpha}_{i}\left(\xi_{i}^{(n)}-\phi_{i}(\bar{u}) \theta_{i}^{(n)}, \eta(x, \bar{u})\right\rangle \geqslant 0\right.
$$


Since $\left.\sum_{i=1}^{p} \bar{\alpha}_{i}\left(f_{i}-\phi_{i}(u) g_{i}\right)\right)+\sum_{i \in I(\bar{u})} \bar{\mu}_{j} h_{j}+\sum_{k \in L} \bar{v}_{k} \ell_{k}$ is strict asymptotic pseudoinvex with respect to $(\eta, D)$ at $\bar{u}$, it follows that

$$
\begin{aligned}
\sum_{i=1}^{p} \bar{\alpha}_{i}\left(f_{i}(\bar{x})-\phi_{i}(\bar{u}) g_{i}(\bar{x})\right) & +\sum_{j \in I(u)} \bar{\mu}_{j} h_{j}(\bar{x})+\sum_{k \in L} \bar{v}_{k} \ell_{k}(\bar{x}) \\
& \left.>\sum_{i=1}^{p} \bar{\alpha}_{i}\left(f_{i}(\bar{u})-\phi_{i}(\bar{u}) g_{i} \bar{u}\right)\right)+\sum_{j \in I(\bar{u})} \bar{\mu}_{j} h_{j}(\bar{u})+\sum_{k \in L} \bar{v}_{k} \ell_{k}(\bar{u}) .
\end{aligned}
$$

But this contradicts (6.17). Hence, $\bar{u}=\bar{x}$.

\section{CONCLUSiON}

Using the results presented in [10], we derived necessary efficiency conditions via convexificators for multiobjective fractional problems involving equality, inequality and set constraints. Under the assumptions on the asymptotic pseudoinvexity for the objective function and the asymptotic quasiinvexity for the constraint functions, the sufficient conditions for weak efficiency were also given together with the theorems of the weak duality, the strong duality, the inverse duality of Wolfe and Mond-Weir types. The results obtained in this paper include the existing results in $[3,6,7]$ as special cases. Note that, in these papers, multiobjective fractional problems have no equality and set constraints. It should be noted that the necessary optimality conditions that are expressed in terms of convexificators, which can be sharper than those expressed via the Clarke, Michel-Penot and Mordukhovich subdifferentials even for locally Lipschitz funtions (see, e.g., [15, Example 2.1]).

\section{Acknowledgements}

The authors would like to thank the referees for their valuable comments and suggestions which improve the paper.

\section{Funding}

This research was funded by Vietnam National Foundation for Science and Technology Development (NAFOSTED) under Grant number 101.01-2017.301.

\section{REFERENCES}

[1] F.H. Clarke, Optimization and Nonsmooth Analysis, Wiley Interscience, New York, 1983.

[2] T.D. Chuong, Optimality and duality for robust multiobjective optimization problems, Nonlinear Anal. 134 (2016), 127-143.

[3] N. Gadhi, Necessary and sufficient optimalty conditions for fractional multi-objective problems, Optimization 57 (2015), 527-537.

[4] X.H. Gong, Scalarization and optimality conditions for vector equilibrium problems, Nonlinear Anal. 73 (2010), 3598-3612.

[5] L.T. Tung, T.T. Khai, P.T. Hung, P.L.B. Ngoc, Karush-Kuhn-Tucker optimality conditions and duality for set optimization problems with mixed constraints, J. Appl. Numer. Optim. 1 (2019), 277-291.

[6] H. Kuk, G.M. Lee, T. Tanino, Optimality and duality for nonsmooth multiobjective fractional programming with generalized invexity, J. Math. Anal. Appl. 262 (2001), 365-375.

[7] Z.A. Liang, H.X. Huang, P.M. Pardalos, Optimality conditions and duality for a class of nonlinear fractional programming problems, J. Optim. Theory Appl. 110 (2001), 611-619.

[8] D.V. Luu, Necessary and sufficient conditions for efficiency via convexificators, J. Optim. Theory Appl. 160 (2014), 510-526. 
[9] D.V. Luu, Convexificators and necessary conditions for efficiency, Optimization 63 (2014), 321-335.

[10] D.V. Luu,Optimality condition for local efficient solutions of vector equilibrium problems via convexificators and applications, J. Optim. Theory Appl. 171 (2016), 643-665.

[11] D.V. Luu, T.T. Mai, Efficiency conditions for multiobjective bilevel programming problems via convexificators, J. Nonlinear Var. Anal. 4 (2020), 399-414.

[12] D.T. Luc, A multiplier rule for multiobjective programming problems with constraints data, SIAM J. Optim. 13 (2002), 168-178.

[13] P. Michel, J.-P, Penot, Calcul sous-différentiel pour des fonctions lipschitziennes et nonlipschitziennes, C. R. Math. Acad. Sci. 12 (1984), 269-272.

[14] B.S. Mordukhovich, Y. Shao, On nonconvex subdifferential calculus in Banach spaces, J. Convex Anal. 2 (1995), 211-228.

[15] V. Jeyakumar, D.T. Luc, Nonsmooth calculus, minimality, and monotonicity of convexificators, J. Optim. Theory Appl. 101 (1999), 599-621.

[16] Z. Hong, L. Jiao, D.S. Kim, On a class of nonsmooth fractional robust multiobjective optimization problems. Part I: Optimality conditions, App. Set-valued Anal Optim. 2 (2020), 109-121.

[17] A.D. Ioffe, Necessary and sufficient conditions for a local minimum. 1: A reduction theorem and first order conditions. SIAM J. Control Optim. 17 (1979), 245-250 